\section{Late Presenting ST Elevation Myocardial Infarction Managed with Adjunctive Laser Therapy}

\section{Abstract}

Here we present a case using Excimer Laser coronary angioplasty (ELCA) prior to ballooning and stent implantation. Laser therapy has been designed to have both a lytic effect on thrombus and a debulking effect on plaque with the idea of reducing distal embolization, microvascular dysfunction and facilitating stent deployment. The technology has been around for over two decades and its use has been explored in acute coronary syndromes, in-stent restenosis, chronic total occlusions and calcified lesions, but its uptake is poor and evidence base mainly limited to registry data. Further, prospective randomized trials are needed in order to better define its role, especially in the Primary $\mathrm{PCl}$ setting.

Keywords: Laser therapy, ST elevation myocardial infarction, Angiography, Angioplasty

Received: November 05, 2015; Accepted: December 11, 2015; Published: December 18,2015

\section{Case Report}

A 63-year-old hypertensive male with no prior history of ischemic heart disease presented to our tertiary hospital in cardiogenic shock with lateral ST elevation on a baseline ECG. The week prior he had been complaining of crescendo angina, which eventually culminated in the onset of rest pain 12 hours prior to hospital presentation.

Immediate angiography was performed and revealed an occluded proximal left circumflex artery (LCX) with a high likelihood of a huge thrombus burden (Figure 1A). The left anterior descending (LAD) was diffusely diseased but unobstructed and the right coronary artery (RCA) was small and non-dominant.

Primary percutaneous coronary intervention $(\mathrm{PCl})$ was performed to the culprit lesion. The obtuse marginal (OM) and LCx were wired and a $0.9 \mathrm{~mm}$ Excimer Laser $\left(60 \mathrm{~mJ} / \mathrm{mm}^{2}\right.$ at 60 pulses/second) was used to dissipate thrombus and ablate plaque. TIMI 3 flow was restored to the vessel and post laser images revealed the proximal $\mathrm{LCX}$ culprit lesion. After $2.5 \mathrm{~mm}$ semi-compliant balloon inflation, optical coherence tomography (OCT) images obtained via pullback method revealed a lipid pool, broken thin cap and was able to identify thrombus (Figure 1B-F). Percutaneous coronary intervention (PCI) was performed with two drug eluting stents (Xience $3.0 \times 38 \mathrm{~mm}$

\author{
Richard J. Jabbour ${ }^{1,2.3}$, \\ Akihito Tanaka ${ }^{1,2}$, \\ Hiroyoshi Kawamoto ${ }^{1,2}$, \\ Azeem Latib ${ }^{1,2}$ and \\ Antonio Colombo ${ }^{1,2}$

\section{Interventional Cardiology Unit, EMO- GVM Centro Cuore Columbus, Milan, Italy \\ 2 Interventional Cardiology Unit, San Raffaele Scientific Institute, Milan, Italy \\ 3 Imperial College, London, UK}

Corresponding author: Richard Jabbour

”rjj03@ic.ac.uk

EMO-GVM Centro Cuore Columbus $48 \mathrm{Via}$ M. Buonarroti, 20145 Milan, Italy.

Tel: +39024812920

Fax: +390248193433

Citation: Jabbour RJ, Tanaka A ,Kawamoto H, et al. Late Presenting ST Elevation Myocardial Infarction Managed with Adjunctive Laser Therapy. Interv Cardiol J 2015, 1:1.

distally and 3.5 x $18 \mathrm{~mm}$ proximally; Abbott Vascular, Santa Clara, CA). An excellent angiographic final result was obtained (Figure 1C).

Here we present a case using Excimer Laser coronary angioplasty (ELCA) prior to ballooning and stent implantation. Now that thrombus aspiration has evaporated from routine use in the primary $\mathrm{PCl}$ setting, the Excimer laser could be useful as it can dissipate thrombus, vaporize pro-coagulant mediators, and may suppress platelet aggregation [1]. Laser therapy also has a debulking effect on plaque and may reduce distal embolization, microvascular dysfunction and facilitate stent deployment [2]. The technology has been around for over two decades and its use has been explored in acute coronary syndromes, in-stent restenosis, chronic total occlusions and calcified lesions, but its uptake has been poor, partly due to the increase cost associated with its use, and evidence base mainly limited to registry data [36]. Further, prospective randomized trials are needed in order to better define its role, especially in the Primary $\mathrm{PCl}$ setting. 


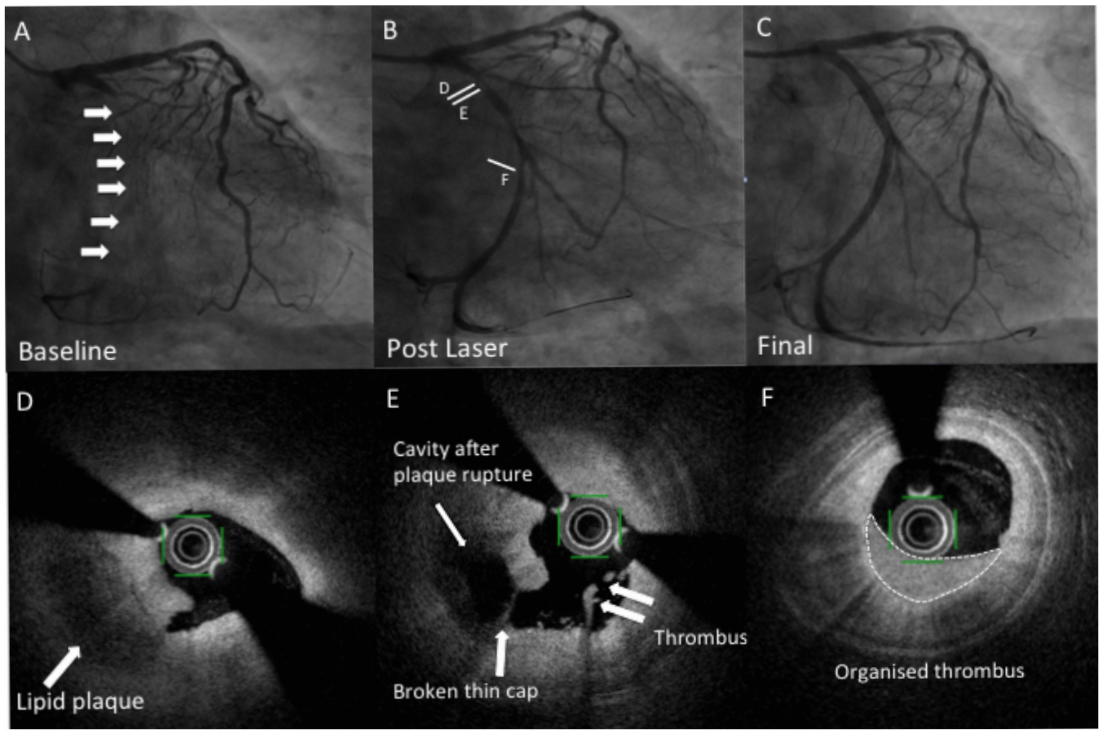

Figure 1 (A) Initial angiogram revealed a proximal circumflex (LCX) culprit lesion; (B) Post Excimer laser angiogram revealing restoration of flow in LCX, letters correspond to relevant OCT Images (D,E,F); (C) Final angiogram after DES implantation; (D) OCT image showing relatively large lipid laden plaque; (E) OCT image revealing site of plaque rupture with thrombus; (F) Mid circumflex OCT image revealing organized thrombus. 


\section{References}

1 Rawlins J, Talwar S, Green M, O'kane P (2014) Optical coherence tomography following percutaneous coronary intervention with Excimer laser coronary atherectomy. Cardiovasc Revasc Med 15: 29-34.

2 Fracassi F, Roberto M, Niccoli G (2013) Current interventional coronary applications of excimer laser. Expert Rev Med Devices 10: 541-549.

3 Ambrosini V, Cioppa A, Salemme L (2008) Excimer laser in acute myocardial infarction: Single centre experience on 66 patients. Int J Cardiol 127: 98-102.
4 Latib A, Takagi K, Chizzola G (2014) Excimer laser Lesion modification to expand non-dilatable stents: The ELLEMENT registry. Cardiovasc Revasc Med 15: 8-12.

5 Shishikura D, Otsuji S, Takiuchi S (2013) Vaporizing thrombus with excimer laser before coronary stenting improves myocardial reperfusion in acute coronary syndrome. Circ J 77: 1445-1452.

6 Dorr M, Vogelgesang D, Hummel A (2007) Excimer laser thrombus elimination for prevention of distal embolization and no-reflow in patients with acute ST elevation myocardial infarction: Results from the randomized LaserAMI study. Int J Cardiol 116: 20-26. 